Original Research Article

\title{
Cupping: a reasonable choice for attenuating morphine withdrawal symptoms in Wistar rats
}

\author{
Parinaz Khosravi ${ }^{1}$, Mohammad M. Roostayi ${ }^{1 *}$, Farideh Dehghan Manshadi ${ }^{1}$, \\ Khosro Khademi-Kalantari', Kambiz Hassanzadeh², Alireza Akbarzadeh Baghban ${ }^{3}$
}

${ }^{1}$ Department of Physiotherapy, Physiotherapy Research Center, School of Rehabilitation, Shahid Beheshti University of Medical Sciences, Tehran, Iran

${ }^{2}$ Department of Physiology and Pharmacology, Cellular and Molecular Research Center, Kurdistan University of Medical Sciences, Sanandaj, Iran ${ }^{3}$ Department of Basic Sciences, School of Rehabilitation, Shahid Beheshti University of Medical Sciences, Tehran, Iran

Received: 19 July 2017

Revised: 02 August 2017

Accepted: 22 August 2017

*Correspondence to:

Dr. Mohammad M. Roostayi, Email: roosta@sbmu.ac.ir

Copyright: (C) the author(s), publisher and licensee Medip Academy. This is an openaccess article distributed under the terms of the Creative Commons Attribution NonCommercial License, which permits unrestricted noncommercial use, distribution, and reproduction in any medium, provided the original work is properly cited.

\begin{abstract}
Background: Nowadays, the sedative drugs are the main treatments to attenuate the opioid withdrawal symptoms. However, physical therapies are found a safe alternative treatment without any serious adverse effects. This study aimed to evaluate the effects of cupping treatment on the morphine withdrawal symptoms in rats.

Methods: Eighty rats were divided into two main groups; treated with morphine or saline, and each group was also divided to cupping and sham cupping subgroups (with single or daily treatment). Injection of morphine and saline were done in the morphine and saline groups twice a day for nine days. On the ninth day, the naloxone was administered and the withdrawal symptoms including jumping, rearing, body grooming, abdominal writing, and wet-dog shaking were recorded for 60 minutes. Cupping or sham cupping was applied on the GV14 once before or daily after withdrawal induction in each group.

Results: The results revealed that a single cupping before the withdrawal induction significantly attenuated the withdrawal symptoms in the morphine group ( $p<0.01)$.But, the daily cupping failed to decrease the withdrawal symptoms in this group. The results also indicated that the daily cupping in the saline group increased the naloxone-induced withdrawal symptoms significantly $(\mathrm{p}<0.01)$.

Conclusions: Single session cupping before the withdrawal induction could decreased the withdrawal symptoms. This finding might be related to the modulation of GABAergic system.
\end{abstract}

Keywor ds: Cupping, Morphine, Withdrawal symptoms

\section{INTRODUCTION}

A major problem with opiates which limit their clinical applications is physical dependence which requires continuation of the drug administration to minimize withdrawal symptom. ${ }^{1}$ Several approaches including administration of drugs such as opioids and benzodiazepines and physical therapy have been developed to prevent physical dependence and minimize withdrawal symptoms. ${ }^{2-4}$ Methadone which compared to opiates has longer half-life, slower addiction liability and higher tolerance is often used in maintenance therapy for opiates addiction. However, the drug has its own risk of dependence especially if the drug administration is discontinued abruptly. The use benzodiazepines to 
attenuate opiate's physical dependency are also as sociated with abuse liability and withdrawal symptoms. ${ }^{5}$

Acupuncture an alternative medicine topic is classified to the needle and non-needle methods including moxibustion, cupping, and acupressure. ${ }^{6}$ Several reports have shown that acupuncture alleviate the chronic pain and withdrawal symptoms in drug abuse..$^{7,8}$ The results of an investigation have shown that the application of needle acupuncture decreased naloxone-precipitated withdrawal symptoms. Lee et al, have reported that the acupuncture at HT7, but not at the control point LI5, decreased significantly the symptoms of the morphine withdrawal and this effect was blocked by the pretreatment with either bicuculline as the GABA (A) receptor antagonist or $\mathrm{SCH}$ 50911 as the GABA (B) receptor antagonist. ${ }^{8}$ On the bas is of results of these investigations it may be concluded that the suppression of the morphine withdrawal symptoms by acupuncture are somewhat mediated by GABA receptors. Also results of another investigation have shown that acupuncture at the specific HT7 points through regulation of GABA receptors modulates the reinforcing effects of the morphine. ${ }^{9}$ Ullah et al. have reported that the acupuncture can stimulate the gates for pain in the CNS which interpret pain sensation, and also elicit the release of morphine like substances (endorphins), serotonin or cortisol which can ultimately lead to pain relief and alter the physiological status of the individual. ${ }^{10}$ According to the literature, the principles of the acupuncture and the acupressure are very similar to that of the cupping therapy. ${ }^{7}$ In most Asian countries, Central Europe and parts of Africa, cupping therapy has been used as a noninvasive physical therapy to reduce the symptoms of many diseases and relieve pain. ${ }^{1-13}$ However, there are many unanswered questions about the biological mechanisms behind the cupping on the drug addiction in humans and animals. In the present study effects of dry cupping on the morphine withdrawal symptoms in rats was investigated.

\section{METHODS}

Eighty male Wistar rats (225-275g) were purchased from the Pasteur Institute (Iran). Six rats per cage were housed $(80 \times 80 \times 40 \mathrm{~cm})$ at the laboratory temperature $\left(20 \pm 3^{\circ} \mathrm{C}\right)$ and $60 \%$ humidity under a 12-h light-dark cycle (lights on at 07:00 A.M). Food (lab chow) and water were also available at libitum. All procedures involving the use of animals were approved by the research committee of the Kurdistan University of Medical Sciences and then were performed according to the Guide for Care and Use of Laboratory Animals (NIH Publication No. 85-23, revised 1985). The drugs which used in this study were Morphine sulfate (Temad Co. Iran) and Naloxone hydrochloride (Darou Pakhsah Co. Iran) that were dissolved in the normal saline.

The equipment for cupping therapy consisted of a plastic cup ( $2.5 \mathrm{~cm}$ diameter), a hand-pump to create negative pressure and a gauge to control the amount of negative pressure (Figure 1). The gauge was calibrated in the centre of control (Yeka Instrumentation Engineering Company) to ensure the reliability and accuracy of the measurement. The results were compared with the Mc-Leod gauge. Based on the results of the previous studies on needle acupuncture. ${ }^{14,15}$ and as it is shown in the images 1 , the GV14 acupuncture point (between C7 and T1 vertebrate) was subjected to cupping (Figure 2).

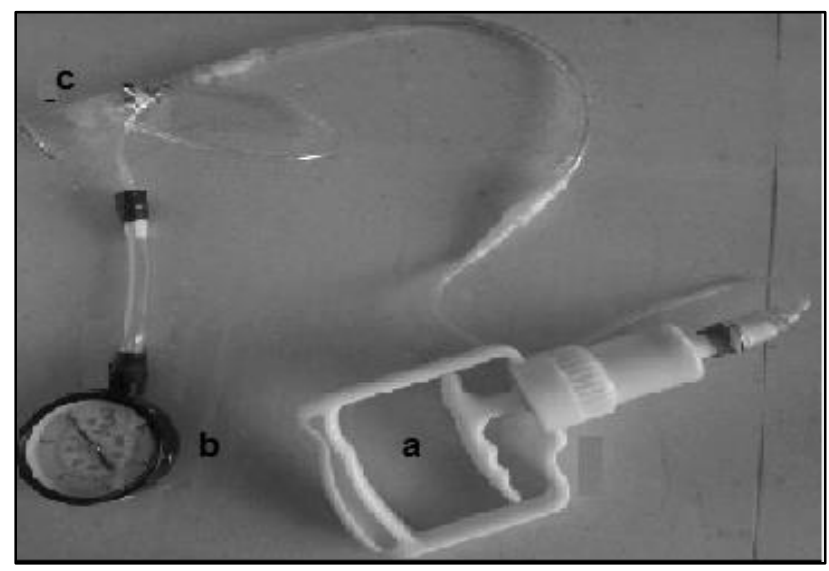

Figure 1: The cupping apparatus: a) The manual suction pump, b) The barometer, c) The cup $(2.5 \mathrm{~cm}$ diameter).

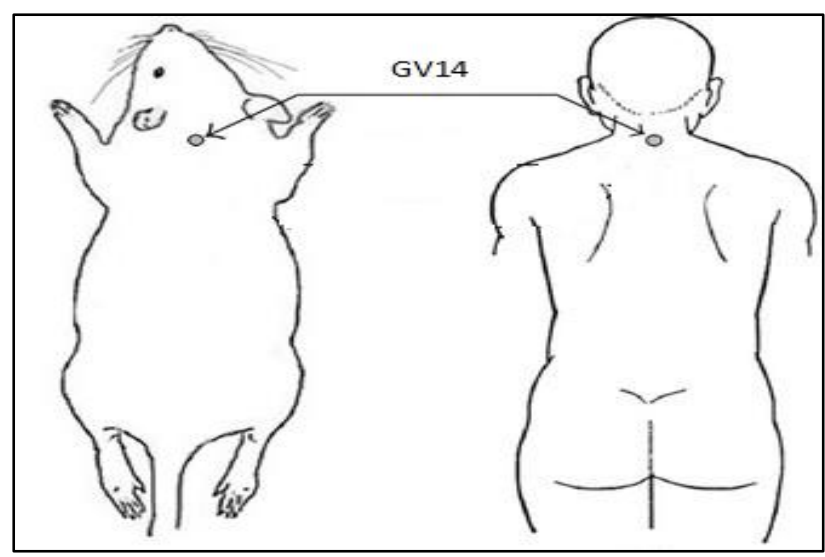

Figure 2: Cupping point. (GV14).

The cupping site was shaved before the experiments. Ultrasonic gel was applied on the skin at the cupping site in order to facilitate the suction. The cupping time was 10 minutes for each session at constant pressure of 200 $\mathrm{mmHg}$. From the results of pilot studies, it was found that this pressure was adequate for desirable suction without any tissue damage.

Rats $(n=80)$ were randomly divided into two main saline and morphine groups and each group was also divided to cupping (+) and sham cupping (-) subgroups. Two different cupping treatment protocols were applied in each subgroup. The rats in the subgroup referred to as daily cup $(+/-)$ were subjected to cupping 10 minutes after injection (saline or morphine) and the rats in the other group referred to as single cup (+/-) were treated just once before 
withdrawal induction. Based on the injection and cupping treatment totally 8 groups were designed as follows:

1. The saline treated sham group: Two groups of rats received saline $(1 \mathrm{~mL} / \mathrm{kg})$ subcutaneously for nine days. The cups were applied without the suction either before the withdrawal induction (single cup (-) group) or daily at 10 minutes after saline injection (daily cup (-) group).

2. The saline treated cupped group: Two groups of rats received the saline $(1 \mathrm{~mL} / \mathrm{kg})$ subcutaneously for nine days. Cupping was applied either before withdrawal induction (single cup (+) group) or daily at 10 minutes after saline injection (daily cup (+) group).

3. The morphine treated sham group: two groups of rats received the additive doses of the morphine subcutaneously for nine days. Cupping was applied either before withdrawal induction (single cup (-) group) or daily at 10 minutes after morphine injection (daily cup (-) group).

4. The morphine treated cupped group: Two groups of rats also received the additive doses of the morphine subcutaneously for nine days. The cupping was done either once before withdrawal induction (single cup $(+)$ group) or daily at 10 minutes after the morphine injection (daily cup (+) group).

For Induction of dependency, additive doses of the morphine were subcutaneously administered twice a day for nine days as follows: Day 1: $5 \mathrm{mg} / \mathrm{kg} / 12 \mathrm{~h}$, Days 2 and 3: $10 \mathrm{mg} / \mathrm{kg} / 12 \mathrm{~h}$, Days 4 and 5: $15 \mathrm{mg} / \mathrm{kg} / 12 \mathrm{~h}$, Day 6 and 7: $20 \mathrm{mg} / \mathrm{kg} / 12 \mathrm{~h}$, and Days 8 and 9: $25 \mathrm{mg} / \mathrm{kg} / 12 \mathrm{~h}$. On the ninth day, only morning dose of the morphine was injected. The method for the additive doses of the morphine was adapted from previous studies. ${ }^{4,16}$

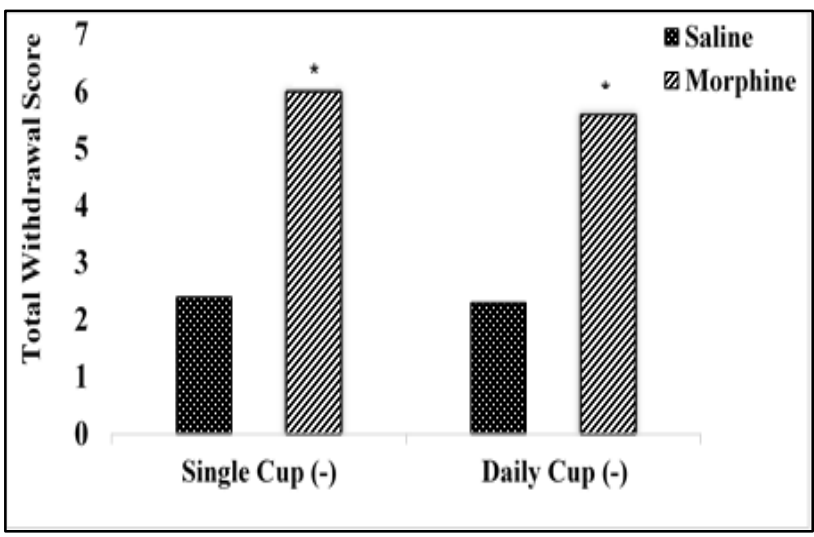

*indicates significant differences $\mathrm{s}$ in comparison with sham cupping in the morphine group $(\mathrm{P}<0.05)$. \# indicates significant differences $\mathrm{s}$ in comparison with sham cupping in the saline group $(\mathrm{P}<0.05)$. Mor $=$ Morphine, $\mathrm{Sal}=$ Saline, $\mathrm{Cup}(-)=$ sham cupping Cup $(+)=$ cupping. The bars indicate means \pm SEM.

Figure 3: A comparis on of the total withdrawal Score (TWS) in the saline and morphine groups after single cupping and single sham cupping.

For induction of the withdrawal symptoms in rat, on the ninth day, 1 hour after injection of the morning dose of the morphine (30 minutes after the cup application with or without suction) rats were treated with naloxone $(4 \mathrm{mg} / \mathrm{kg}$, ip) by injection. The chosen dose for naloxone was adapted from the previous studies. ${ }^{4}$ The behavioral assessment of the opioid withdrawal signs including jumping, rearing, body grooming, abdomen writhing, and wet-dog shaking was started exactly one hour after the naloxone injection. For this purpose the rats were examined individually in a clear Plexiglas chamber $(50 \mathrm{~cm} \times 25 \mathrm{~cm} \times 15 \mathrm{~cm})$ which was placed in other dark chamber to avoid the environmental perturbations. A digital camera connected to a recording computer was placed on the inner chamber to show rats' behaviors simultaneously. Reactions of rats were evaluated by the same observer who was not aware of the nature of the treatment received by each animal. ${ }^{4}$ In this study other than reporting the withdrawal symptoms, total withdrawal score for each animal was also calculated because there were significant differences in withdrawal signs and specific behaviors of individual animals under different conditions. ${ }^{17}$ To achieve this goal, 5 assessed signs in an animal were scored on the bas is of the division of absolute frequency of each behavior into its respective weighting factor (Table 1) and then scores of the withdrawal signs were added.

\section{Table 1: Weighing factor of different} withdrawal signs.

\begin{tabular}{|ll|}
\hline Behavior signs & Weighing factor \\
\hline Jumping & 4 \\
\hline Wet-Dog Shake & 5 \\
\hline Abdomen Writhing & 5 \\
\hline Body Grooming & 10 \\
\hline Rearing & 20 \\
\hline
\end{tabular}

Before and after cupping a locomotor activity of each animal was assessed through direct observation using an interval scale that measures the frequency of crossing the painted lines on the underside floor of the Plexiglas behavioral arena. ${ }^{18}$

\section{Analyses of data}

Statistical comparisons between experimental groups were made by a one-way ANOVA test followed by a Tukey post-hoc test for pairwise comparisons. All results were shown as the mean \pm SEM with the statistical significance set at $\mathrm{p}<0.05$.

\section{RESULTS}

\section{Confirmation of dependency and naloxone-induced withdrawal}

Results showed that the withdrawal signs including jumping, rearing, body grooming, abdomen writhing, and wet-dog shaking were significantly greater in the morphine groups than the saline groups. Also the data analysis revealed that there was a significant $(\mathrm{P}<0.001)$ 
difference in the Total Withdrawal Score (TWS) between the saline and the morphine administrated animals in both single cup (-) group and daily cup (-) group; this means that the animals which received the morphine became dependent (Figure 3).

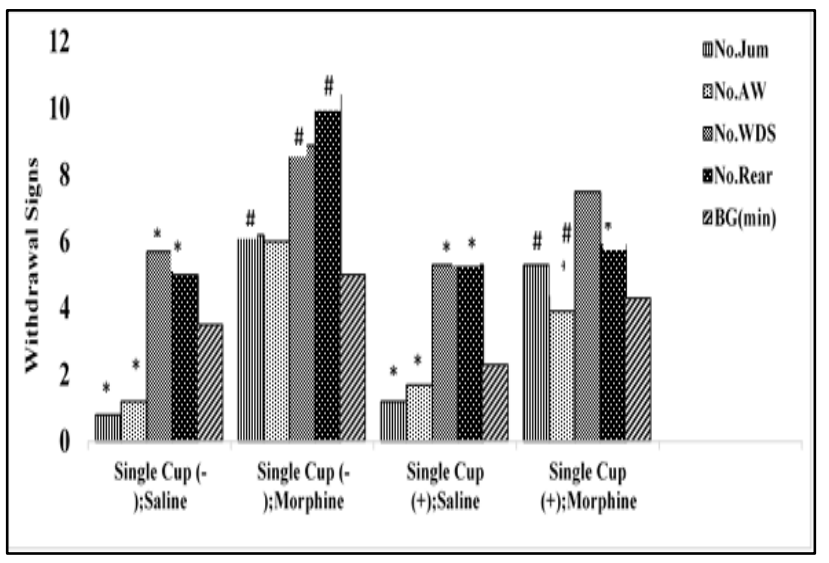

*indicates significant differences between sham cupping in the morphine group $(\mathrm{P}<0.05)$. \# indicates significant differences between in comparison with saline group $(\mathrm{P}<0.05)$. The bars indicate means \pm SEM . Jum $=$ Jumping, AW = Abdomen Writing, WDS $=$ Wet-dog shake, Rear $=$ Rearing, BG $=$ Body Grooming, Mor $=$ Morphine, Sal $=$ Saline, Cup $(-)=$ sham cuping, Cup $(+)$ $=$ Cupping.

Figure 4: The effects of single cupping on the naloxone-precipitated withdrawal signs in the morphine and saline treated groups.

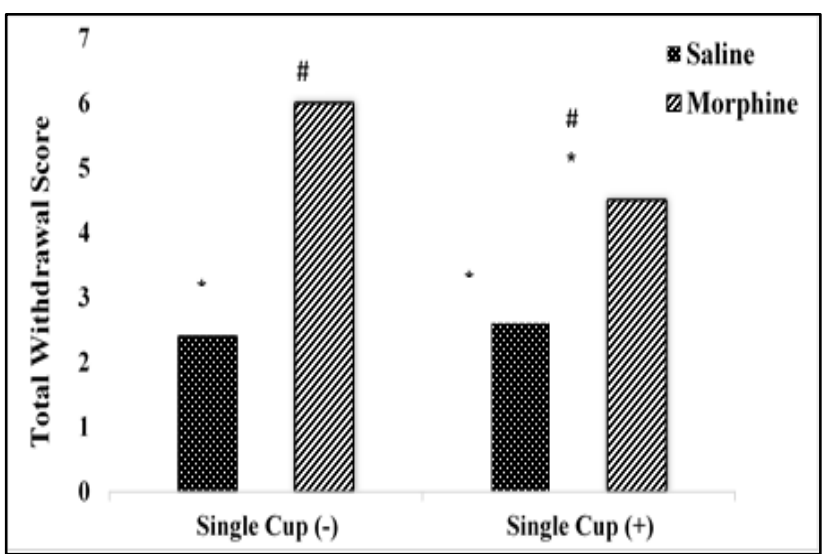

It indicates significant differences compared between these groups $(\mathrm{P}<0.05)$. Mor $=$ Morphine, Sal $=$ Saline, Single Cup $(-)$ $=$ Single sham cupping, Daily Cup $(-)=$ Daily sham cupping. The bars indicate means \pm SEM

Figure 5: A comparis on of the Total Withdrawal Score (TWS) in the saline and morphine groups after both single and daily sham cupping.

\section{Effect of single cupping}

Results shown in Figure 4 revealed that before withdrawal induction, single ses sion cupping decreased all withdrawal signs in the morphine group. However compared to saline group, attenuation was statistically significant only in abdomen writing $(\mathrm{p}<0.05)$ and rearing $(\mathrm{p}<0.001)$. Results also indicated that there was no significant difference between cupping (+) and sham cupping $(-)$ in saline treated groups.

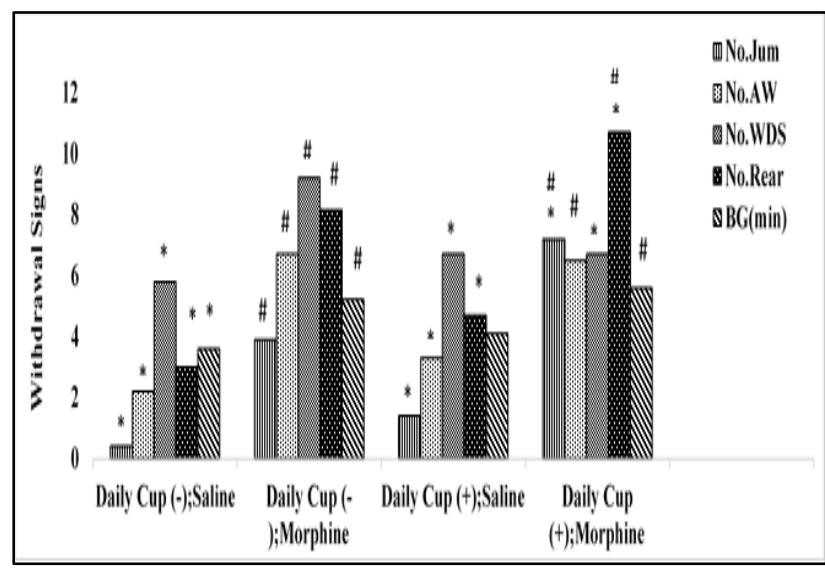

*indicates significant differences in comparison with sham cupping in the morphine group $(\mathrm{P}<0.05)$. \# indicates significant difference $\mathrm{s}$ in comparison with sham cupping in the saline group $(\mathrm{P}<0.05)$. The bars indicate means \pm SEM. Jum $=$ Jumping, AW = Abdomen Writing, WDS = Wet - Dog Shake, Rear = Rearing, $\mathrm{BG}=$ Body Grooming, Mor $=$ Morphine, Sal $=$ Saline, Cup $(-)=$ sham cupping, $\operatorname{Cup}(+)=$ Cupping.

Figure 6: Fffects of the daily cupping on the naloxoneprecipitated withdrawal signs in the morphine and saline groups.

Furthermore, as it is shown in Figure 5, there was a significant decrease in the TWS in the cupping (+) compared to the sham cupping (-) group in morphine treated rats $(\mathrm{p}<0.01)$.

\section{Effects of daily cupping}

Figure 6 depicts the results of the daily cupping on the withdrawal symptoms in morphine and saline groups. In the morphine group, the results revealed that the daily cupping $(+)$ increased jumping $(\mathrm{p}<0.001)$ and rearing $(\mathrm{p}<0.05)$, and conversely decreased the wet-dog shaking $(\mathrm{p}<0.01)$ compared to the sham cupping (-) treated rats. In addition, the other two symptoms (abdomen writing and body grooming) seemed to be unchanged. As you see in Figure 6, the daily cupping (+) did not change the TWS in the morphine group, whereas there was a different behavior in cupping (+) and sham cupping (-) in the saline group after daily cupping; the daily cupping (+) led to an increase in the TWS in comparison with the sham cupping $(-)$ treated rats $(\mathrm{p}<0.01)$.

\section{DISCUSSION}

Different drugs and strategies have been investigated to find an effective way for preventing the opioid dependence and the withdrawal symptoms. Although the exact mechanisms of mediating these effects have not firmly been identified yet, certain changes in catecholaminergic, serotonergic, cholinergic, and GABAergic or peptidergic 
transmission are reported during the chronic opiate administration. ${ }^{1}$

The results of this study confirmed those of the previous studies in that the chronic morphine adminis tration and the naloxone injection lead to the dependency and the withdrawal symptoms, respectively. ${ }^{19}$ The results revealed that a single session cupping (+) before the withdrawal induction significantly attenuated the withdrawal symptoms. According to our findings, the daily cupping $(+)$ however, failed to decrease the withdrawal symptoms in the morphine group, the daily cupping (+) in the saline group increased the naloxone-induced withdrawal symptoms significantly. This result suggests that the daily cupping has developed the morphine dependence probably through the endogen opioid release activation.

The effect of cupping on the morphine withdrawal symptoms has rarely been investigated. The results of this study were in line with the findings of other studies in that the physical therapy such as the acupuncture attenuated the morphine self-administration, and the naloxone precipitated the withdrawal symptoms in the rats. Their results suggest that the acupuncture effect on the morphine dependency is through the GABAergic system activation and recovering the dopamine depletion during the morphine withdrawal. ${ }^{8,9}$ In addition, there are several lines of evidences indicating that the cupping reduces the pain conditions.$^{20}$ In this respect, the reductions in the pain scores are attributed to the sound rationale since the cupping therapy can elicit the release of the morphine-like substances (endorphins), and serotonin or cortisol that can ultimately lead to the pain relief and alter the physiological status of the individual. ${ }^{21}$ In additions, it is believed that the stimulation of the cupping points leads to the pain gates to be overwhelmed by increasing the frequency of the impulses, and resulting in the closure of the gates and the pain reduction. ${ }^{22}$

According to the present study, the alleviative effects of cupping on morphine withdrawal was not the result of reduction in motor activity in rats since results of locomotor activity test showed no change in rats' motor performance. Finally, because of different adverse effects of the drugs, such as opioids and benzodiazepines, which are used for the prevention of the withdrawal syndrome and their capability of inducing the dependency, the use of the physical treatment (such as cupping) as an adjunct therapy probably allows physicians to reduce the administration of drugs.

\section{CONCLUSION}

A single cupping before the morphine withdrawal induction decreased the naloxone-precipitated withdrawal symptom in rats. This finding might be related to the effects of the cupping on pain control through the release of endogen opioids and modulation of GABAergic system However more investigations are required to clarify this effect and its mechanism.

\section{ACKNOWLEDGEMENTS}

The authors are thankful to the Research Centre of the Rehabilitation School, Shahid Beheshti University of Medical Sciences and all the colleagues and students helped us to do this study.

Funding: No funding sources

Conflict of interest: None declared

Ethical approval: The study was approved by the Institutional Ethics Committee of the Kurdistan University of Medical Sciences and to the Guide for Care and Use of Laboratory Animals (NIH Publication No. 85-23, revised 1985)

\section{REFERENCES}

1. Rang H, Dale M, Ritter J. Rang \& Dale's Pharmacology. $7^{\text {th }}$ Ed. London: University College London; 2011.

2. Habibi As1B, Hassanzadeh K, Khezri E, Mohammadi S. Evaluation the effects of dextromethorphanand midazolam on morphine induced tolerance and dependence in mice. Pak J Biol Sci.2008;11(13):16905.

3. Chen Y, Sommer C. The role of mitogen-activated protein kinase (MAPK) in morphine tolerance and dependence. Mol Neurobiol. 2009;(40):101-7.

4. Habibi As1B, Hassanzadeh K. Effects of ketamine and midazolam on morphine induced dependence and tolerance in mice. DARU. 2004;(12):101-5.

5. Katzung B. Basic and clinical pharmacology, chapter 31. 12 ${ }^{\text {th }}$ Ed. New York: Mc Graw-hill; 2012.

6. Chen CJ, Yu HS. Acupuncture, electrostimulation, and reflex therapy in dermatology. Dermatol Ther. 2003;16(2):87-92.

7. Hssanien MMR, Mansoura Fawaz S, Ahmed AF, Al Emadi S. Effect of cupping therapy in treating chronic headache and chronic back pain at Al heijamah clinic HMC. World Family Med J. 2010;8(3):30-6.

8. Lee JH, Kim HY, Jang EY, Choi SH, Han CH, Lee $\mathrm{BH}$, et al. Effect of acupuncture on naloxoneprecipitated withdrawal syndrome in morphineexperienced rats: the mediation of GABA receptors. Neurosci Lett. 2011;504(3):301-5.

9. Yoon SS, Kim H, Choi KH, Lee BH, Lee YK, Lim SC, et al. Acupuncture suppresses morphine selfadministration through the GABA receptors. Brain Res Bull. 2010;81(6):625-30.

10. Ullah K, Younis A, Wali M. An investigation into the effect of Cupping Therapy as a treatment for Anterior Knee Pain and its potential role in Health Promotion. The Internet Journal of Alternative Medicine. 2006;4(1).

11. Roostayi MM, Bagheri H, Talebian MS, Firooznia K, Razi M, Hosseini M, et al. The effects of vacuumic bracing system on the patellofemoral articulation in patients with patellofemoral pain syndrome Complement. Ther Clin Pract. 2009;15(1):29-34. 
12. Kim JI, Lee MS, Lee DH, Boddy K, Ernst E. Cupping for Treating Pain: A Systematic Review. Evidence Based Complementary and Alternative Medicine; 2009.

13. Roostayi MM, Norouzali T, Dehghan MF, Akbarzadeh BA. The Effects of Cupping Therapy on Skin's Biomechanical Properties in Wistar Rats. Chinese Medicine. 2016;(7):25-30.

14. Kim ST, Moon W, Chae Y, Kim YJ, Lee H, Park HJ. The effect of electroaucpuncture for 1-methyl-4phenyl-1,2,3,6-tetrahydropyridine-induced proteomic changes in the mouse striatum. J Physiol Sci. 2010;(60):27-34.

15. Xu YD, Cui JM, Wang Y, Yin LM, Gao CK, Liu XY, et al. Proteomic analysis reveals the deregulation of inflammation- related proteins in acupuncture-treated rats with asthma onset. Evid Based Complement Alternat Med. 2012.

16. Habibi Asl B, Sadegh amiri O, Charkhpour M, Hasanzadeh K. A novel pharmacological role for minocycline: attenuating the withdrawal syndrome of morphine in rat. Pharmaceutical Sciences. 2009;(15):67-74.

17. Riahi E, Mirzaii DI, Karimian SM, Sadeghipour Roodsari HR, Dehpour AR. Attenuation of morphine withdrawal signs by a GABAB receptoragonist in the locus coeruleus of rats. Behav Brain Res. 2009;196(1):11-4.

18. Crawley JN, Gerfen GR, Rogawski MA, Sibley DR, Skolnick P, Wray S. Short protocols in neuroscience. John Wiley \& Sons Inc. 2007.

19. Charkhpour M, Nayebi AM. Evaluation of the role of 5-HT2 receptors in dorsal and medianraphe nuclei on the morphine withdrawal syndrome in rat. Pharmaceutical Sciences. 2006;(12):33-40.

20. Michalsen A, Bock S, ludtke R. Effectiveness of cupping therapy in brachialgia paraestetica nocturna: results of a randomized controlled trial. Forsch Komplementarmed. 2007;14:19.

21. Schulte E. Complementary therapies: Acupuncture: Where East meets west. Res Nurs. 1996;59:55-7.

22. Cadwell V. A primer on acupuncture. J Emerg Nurs. 1998;(24):514-7.

Cite this article as: Khosravi $\mathrm{P}$, Roostayi MM, ManshadiFD, Khosro KK, Kambiz H, Baghban AA. Cupping: a reasonable choice for attenuating morphine withdrawal symptoms in Wistar rats. Int $\mathbf{J}$ Basic Clin Pharmacol 2017;6:2783-8. 